\title{
Clinical and Laboratory Findings in Patients with Acute Respiratory Symptoms that Suggest the Necessity of Chest X-ray for Community- Acquired Pneumonia
}

\author{
Azadeh Ebrahimzadeh ${ }^{1}$; Mahyar Mohammadifard ${ }^{2,}$; Godratallah Naseh ${ }^{3}$; Alireza \\ Mirgholami $^{2}$ \\ ${ }^{1}$ Department of Internal Medicine, Faculty of Medicine, Birjand University of Medical Sciences, Birjand, Iran \\ ${ }_{3}^{2}$ Department of Radiology, Imam Reza Hospital, Birjand University of Medical Sciences, Birjand, Iran \\ ${ }^{3}$ Department of Surgery, Imam Reza Hospital, Birjand University of Medical Sciences, Birjand, Iran \\ ${ }^{*}$ Corresponding author: Mahyar Mohammadifard, Department of Radiology, Imam Reza Hospital, Birjand University of Medical Sciences, Birjand, Iran. Tel: +98-5118414499, Fax: +98- \\ 5612226898, E-mail: mahyar.mohammadifard@yahoo.com
}

Received: July 12, 2013; Revised: April 9, 2014; Accepted: April 15, 2014

\begin{abstract}
Background: Pneumonia is a common illness in all parts of the world and is considered as a major cause of death among all age groups. Nevertheless, only about $5 \%$ of patients referring to their primary care physicians with acute respiratory symptoms will develop pneumonia.

Objectives: This study was performed to derive practical criteria for performing chest radiographs for the evaluation of communityacquired pneumonia (CAP).

Patients and Methods: A total of 420 patients with acute respiratory symptoms and positive findings on chest radiograph were evaluated from December 2008 to December 2009. The subjects were referred to outpatient clinics or emergency departments of Birjand's medical university hospitals, Iran, and were enrolled as positive cases. A checklist was completed for each patient including their demographic information, clinical signs and symptoms (cough, sputum production, dyspnea, chest pain, fever, tachycardia, and tachypnea), abnormal findings in pulmonary auscultation and laboratory findings (erythrocyte sedimentation rate, C-reactive protein levels, and white blood cell count). An equal number of age-matched individuals with acute respiratory symptoms, but insignificant findings on chest radiography, were included as the control group. Finally, the diagnostic values of different findings were compared.

Results: The data showed that vital signs and physical examination findings are useful screening parameters for predicting chest radiograph findings in outpatient settings. Therefore, by implementing a prediction rule, we would be able to determine which patients would benefit from a chest X-Ray (sensitivity, $94 \%$ and specificity, $57 \%$ ).

Conclusion: This study's findings suggest that requesting chest radiographs might not be necessary in patients with acute respiratory symptoms unless the vital signs and/or physical examination findings are abnormal. Considering the $94 \%$ sensitivity of this rule for predicting CAP, a chest radiograph is required for patients with unreliable follow-ups or moderate to high likelihood of morbidity if CAP is not initially detected.
\end{abstract}

Keywords: Pneumonia; Mass Chest X-Ray; Prediction Rule; Acute Chest Syndrome

\section{Background}

Pneumonia is a common illness all around the world and is considered as a major cause of death among all age groups (1). In Iran, pneumonia is the most common cause of hospitalization in the elderly and patients with primary antibody deficiencies $(2,3)$. Nonetheless, only $5 \%$ and $28 \%$ of patients with acute respiratory symptoms who are referred respectively to primary care physicians (4) and emergency departments (EDs) (5) develop pneumonia. According to most clinical guidelines, the gold standard tool for diagnosing pneumonia is a chest X-ray (CXR), which can distinguish this condition from other respiratory tract infections (6). However, many clinicians might only rely on the patient's medical history and physical examination findings to diagnose or exclude pneumonia.
Community-acquired pneumonia (CAP) is the most common type of pneumonia. Despite frequent use of $\mathrm{X}$ ray in clinical settings, there are still debates regarding its necessity in patients suspected of having CAP. In this regard, Ayalon et al. investigated the role of physical examination in the diagnosis of pneumonia and demonstrated the significant benefit of CXR as an ancillary test for the diagnosis of pneumonia in ED settings (7). In addition, according to a review published in 2003, CXR was considered as the gold standard for diagnosing pneumonia. Researchers doubted that the decision to perform CXR should solely rely on experts' opinions, which are based on strategies for optimizing the balance between harms and benefits. Consequently, they recommended

Copyright (C) 2015, Tehran University of Medical Sciences and Iranian Society of Radiology. This is an open-access article distributed under the terms of the Creative Commons Attribution-NonCommercial 4.0 International License (http://creativecommons.org/licenses/by-nc/4.0/) which permits copy and redistribute the material just in noncommercial usages, provided the original work is properly cited. 
a combination of the patient's medical history, physical examination, and laboratory data to estimate short-term risks and to determine the appropriate treatment (8).

\section{Objectives}

Although several investigators have attempted to provide prediction rules to improve the detection of CAP in outpatient settings during the past three decades, there have been contradictory conclusions in this regard (4, 9-17). Accordingly, the aim of the present study was to evaluate the predictive value of the eight most common clinical parameters and three most frequent laboratory variables in patients presenting with acute respiratory symptoms. Therefore, the subjects were divided into two large groups of 420 to determine the effect of clinical and laboratory data for X-ray-based diagnosis. The groups were as follows: the case group included those with positive CXR findings and the control group consisted of subjects with insignificant CXR findings.

\section{Patients and Methods}

In this case-control study, all adult patients (18 years of age or older) with acute respiratory symptoms (one or more of the following symptoms: cough, sputum, dyspnea, and chest pain) and positive findings on CXR were included as positive cases $(n=420)$. The subjects had been referred to outpatient clinics or EDs of medical university hospitals in Birjand, Iran, from December 2008 to December 2009. Positive findings on CXR were defined as the presence of new consolidations without an air bronchogram, pleural effusion, abscess or empyema in the setting of acute respiratory symptoms. Patients with subtle airspace opacities (equivocal findings) were also considered as positive cases. An equal number of cases $(n=420)$ with acute respiratory symptoms but insignificant findings on CXR were recruited as the control group; the subjects were matched in terms of age and the date of the CXR procedure. Those with findings suggestive of hospital-acquired pneumonia (positive findings on CXR within ten days of discharge), aspiration pneumonia, and those who had recently received antibiotics or steroids were excluded.

An infectious disease specialist examined all patients and the data regarding clinical symptoms and signs were recorded. Afterwards, CXRs were performed and the clinician was blinded to the radiographic results. A boardcertified radiologist, unaware of the clinical findings, interpreted all CXRs. The clinical indicators included cough, sputum production, dyspnea, chest pain, fever (body temperature $\geq 38^{\circ} \mathrm{C}$ ), tachycardia (heart rate $\geq$ 100 beats per minute), tachypnea (respiratory rate $\geq 20$ breaths per minute), and abnormal findings on pulmonary auscultation and examination (crackles, decreased breath sounds, dullness on percussion, and rhonchi). The erythrocyte sedimentation rate (ESR), C-reactive protein (CRP) levels, and white blood cell (WBC) count were measured for all patients. Abnormal values of these labo- ratory tests were defined as follows: ESR (mm/h) higher than the males' age divided by two or females' age plus ten divided by two (16), CRP $>5 \mathrm{mg} / \mathrm{L}(47.62 \mathrm{mmol} / \mathrm{L})$, and $\mathrm{WBC}>10000 / \mu \mathrm{L}$ or $<4000 / \mu \mathrm{L}$.

The sensitivity and specificity of all clinical indicators were calculated using the IBM SPSS 19 software for Windows (IBM Corp., IBM SPSS Statistics for Windows, Armonk, NY: IBM Corp.); odds ratios (OR) were also calculated for each of the indicators. Additional indicators were defined as "any abnormal findings in vital sign assessment" (positive test results = fever, tachycardia, and/ or tachypnea), "any abnormal findings in vital sign assessment or physical examination" (positive test results = fever, tachycardia, tachypnea, and/or abnormal findings on pulmonary examination) and "any abnormal findings in laboratory test" (positive test results = abnormal values of ESR, CRP, or WBC). According to the analyses, we attempted to provide a prediction rule to determine which patients would benefit from CXR. The diagnostic accuracy of this rule was determined regarding the area under the receiver operating characteristic (ROC) curve.

\section{Results}

During the 12-month study period, 420 patients with acute respiratory symptoms and positive findings on CXR were eligible for participating in the study. An equal number of individuals were selected as the control group with similar demographic characteristics as the case group (Table 1). The analyzed data are presented in Table 2 . The clinical symptoms and signs were compared, and as listed in Table 2, there was a significant difference between the two groups regarding clinical indicators including fever, tachycardia and tachypnea (abnormal vital signs). Similarly, the difference between the two groups in terms of pulmonary auscultation according to physical examination was statistically significant. With regards to the aims of this study, the sensitivity and specificity (95\% confidence interval) of the statistically significant variables were calculated (Table 2). The clinical indicator with the greatest sensitivity for pneumonia (diagnosed by CXR) was cough, with a sensitivity of $89.5 \%$.

The presence of "any abnormal findings in vital sign assessment", "any abnormal findings in vital sign assessment or physical examination", and "any abnormal findings in laboratory test" was studied, and these indicators were compared according to the recorded data (Table 2). All three of these new "combined indicators" exhibited higher prevalence in patients with CAP (positive findings on CXR in the setting of acute respiratory symptoms) in comparison with the controls. However, only for one parameter (any abnormal findings in vital sign assessment or physical examination), the sensitivity (94.0\%), specificity (57.4\%), and OR (23.1) were optimal for being used as a screening parameter. This combined prediction rule had an area under the ROC of 0.751 , which suggested that it was a relatively effective rule with $75 \%$ accuracy and $94 \%$ 
Ebrahimzadeh A et al.

Table 1. Demographic Data of the Patients Presenting Acute Respiratory Symptoms ${ }^{\mathrm{a}, \mathrm{b}}$

\begin{tabular}{lcc}
\hline Variables & Case Group (No. $=\mathbf{4 2 0})$ & Control Group (No. $=\mathbf{4 2 0})$ \\
\hline Mean Age, $y$ & 60 & 63 \\
Age Range, $y$ & $18-87$ & $19-86$ \\
Gender & & \\
$\quad$ Male & $220(52.4)$ & $227(54.0)$ \\
Female & $200(47.6)$ & $193(46.0)$ \\
Underlying Lung Disease $^{c}$ & $50(11.9)$ & $28(6.7)$ \\
\hline
\end{tabular}

a Data are presented as No. (\%) or Mean.

$\mathrm{b}$ None of these variables showed a significant difference between case and control groups, as indicated by the independent-samples $\mathrm{t}$ test and Chi square test $(\mathrm{P}>0.05)$.

${ }^{\mathrm{C}}$ Underlying lung disease is defined as chronic obstructive disease, interstitial lung disease, or cancer.

Table 2. Analysis of Clinical and Laboratory Indicators in Patients with Acute Respiratory Symptoms a

\begin{tabular}{|c|c|c|c|c|c|}
\hline Indicators & Cases $(n=420)^{b}$ & Controls $(n=420)^{b}$ & $\begin{array}{l}\text { Sensitivity }{ }^{\mathrm{C}} \\
(95 \% \mathrm{CI})\end{array}$ & $\begin{array}{l}\text { Specificity }^{\mathrm{C}} \\
(95 \% \mathrm{CI})\end{array}$ & $\begin{array}{l}\text { Odds Ratio } \\
(95 \% \mathrm{CI})\end{array}$ \\
\hline Cough & $376(89.5)$ & $272(64.8)$ & $89.5(86.7-91.5)$ & $35.5(30.4-40.8)$ & $4.6(2.9-6.7)$ \\
\hline Sputum & $354(84.3)$ & $168(40.0)$ & $84.3(80.6-87.0)$ & $60.0(55.4-64.7)$ & $8.0(5.6-10.4)$ \\
\hline Dyspnea & $323(76.9)$ & $180(42.9)$ & $76.9(72.6-80.4)$ & $57.1(60.5-70.2)$ & $4.4(2.7-6.5)$ \\
\hline Chest pain & $315(75.0)$ & $201(47.9)$ & $75.0(71.1-79.0)$ & $52.1(46.3-57.3)$ & $3.5(2.4-5.1)$ \\
\hline Fever $^{d}$ & $285(67.9)$ & $68(16.2)$ & $67.9(62.9-73.2)$ & $83.8(80.1-86.5)$ & $7.0(4.8-10.2)$ \\
\hline Tachycardia ${ }^{\mathrm{d}}$ & $231(55.0)$ & $63(15.0)$ & $55.0(49.6-59.4)$ & $85.0(81.0-88.4)$ & $6.9(4.7-10.1)$ \\
\hline Tachypnea $^{\mathrm{d}}$ & $252(60.0)$ & $44(10.5)$ & $60.0(55.3-64.9)$ & $89.5(86.5-91.5)$ & $12.8(7.6-17.4)$ \\
\hline $\begin{array}{l}\text { Abnormal Auscultation } \\
\text { Findings } \mathrm{e}^{\mathrm{a}}\end{array}$ & $370(88.1)$ & $133(31.7)$ & $88.1(85.1-90.3)$ & $68.3(63.3-73.6)$ & $16.0(11.2-20.8)$ \\
\hline Elevated ESR & $119(28.3)$ & $55(13.1)$ & $28.3(22.3-33.6)$ & $86.9(82.8-90.3)$ & $7.2(5.1-10.3)$ \\
\hline Elevated CRP & $168(40.0)$ & $22(5.2)$ & $40.0(34.7-45.4)$ & $94.6(91.8-96.7)$ & $12.7(7.5-17.3)$ \\
\hline Abnormal WBC & $204(48.6)$ & $94(22.4)$ & $48.6(42.7-54.0)$ & $77.6(73.4-81.1)$ & $3.3(2.2-4.9)$ \\
\hline $\begin{array}{l}\text { Any Abnormal Findings } \\
\text { in VS }\end{array}$ & $361(86.0)$ & $118(28.1)$ & $86.0(81.9-89.4)$ & $77.9(73.781 .5)$ & $9.5(7.2-12.8)$ \\
\hline $\begin{array}{l}\text { Any Abnormal Findings in } \\
\text { VS or PE }\end{array}$ & $395(94.0)$ & $179(42.6)$ & $94.0(91.1-96.3)$ & $57.4(60.8-70.5)$ & $23.1(12.7-43.1)$ \\
\hline $\begin{array}{l}\text { Any Abnormal Findings } \\
\text { in LAB }\end{array}$ & $254(60.5)$ & $110(26.2)$ & $60.5(55.2-65.5)$ & $73.8(69.8-77.7)$ & $6.8(4.5-10.0)$ \\
\hline \multicolumn{6}{|c|}{$\begin{array}{l}\text { a Abbreviations: CI, confidence interval; CRP, C-reactive protein; ESR, erythrocyte sedimentation rate; LAB, laboratory tests (ESR, CRP, or WBC); PE, } \\
\text { physical examination (pulmonary auscultation); and VS, vital sign (body temperature, heart rate, and respiratory rate). } \\
\text { b Data are presented as No. (\%). } \\
\text { c Data are presented as (\%). } \\
\text { d Body temperature } \geq 38^{\circ} \text { C; heart rate } \geq 100 \text { beats per minute; respiratory rate } \geq 20 \text { breaths per minute. } \\
\text { e Presence of crackles, decreased breath sounds, dullness on percussion, or rhonchi. }\end{array}$} \\
\hline
\end{tabular}

sensitivity. Table 2 lists the sensitivity and specificity of other clinical, laboratory, and "combined" indicators. Elevated CRP alone had a specificity of $94.6 \%$. The sensitivity of elevated ESR was $28.3 \%$, whereas its specificity was $86.9 \%$.

\section{Discussion}

The results of the present study revealed that vital signs and physical examinations are reliable parameters for predicting chest radiograph findings in outpatient settings; thus, it is possible to determine which patients would benefit from CXR. Performing CXR, only in patients with acute respiratory symptoms and abnormal findings in vital signs assessment and physical examinations, enabled us to correctly identify $94 \%$ of patients with positive findings on CXR (positive cases) and avoided unnecessary CXRs in 57\% of the patients with insignificant findings on CXR (controls); this indicates 94\% sensitivity and $57 \%$ specificity of the prediction rule.

The suggested rule indicates that performing CXRs would be unnecessary in patients with acute respiratory symptoms (cough, sputum, dyspnea or chest pain), who 
present normal findings in vital signs assessment (body temperature, heart rate, and respiratory rate) and physical examination (lung auscultation). Our findings are consistent with those of Gennis et al. (11) and O'Brien et al. (12), who provided similar guidelines and prediction rules for ordering CXR, using clinical criteria for the detection of pneumonia in adults. However, our results differed from the findings of Singal et al. (10) and Butcher et al. (18), who demonstrated the inefficiency of clinical findings as screening parameters, and Graffelman et al. (13), who concluded that models based only on clinical findings do not reliably predict the presence of pneumonia.

According to the guidelines of the American Thoracic Society (ATS) for the management of CAP (2007), a CXR is required for the routine evaluation of patients who are likely to have pneumonia, in order to establish a diagnosis and help differentiate CAP from other common causes of cough and fever such as acute bronchitis (19). In Iran, there are no national guidelines for CAP management. Most of the clinicians in Iran (and in other countries such as the United States) (10) do not follow the aforementioned guidelines and mainly rely on their personal experiences. They often do not order a CXR for patients with suspected CAP, unless the vital signs and/or physical examination findings are abnormal. Our findings supported these practices by providing objective data.

The British Thoracic Society (BTS) guidelines for the management of CAP in adults (updated in 2009) provide recommendations, which are more similar to the present prediction rule than to the ATS guidelines. According to BTS guidelines, all patients who are admitted to hospitals with signs and symptoms suggestive of CAP should undergo a CXR as soon as possible to confirm or refute the diagnosis. However, it is stated that in non-emergent outpatient settings, it is not necessary to perform CXR in patients with signs and symptoms suggestive of CAP, unless the following conditions are present:

1) The diagnosis is doubted and a CXR can help with the differential diagnosis and the management of the acute illness.

2) The progress following the treatment for suspected CAP is not satisfactory at follow-up.

3) The patient is at risk of underlying lung pathologies such as lung cancer (6).

By implementing this prediction rule in our study, it was shown than CXR findings were insignificant in $57 \%$ of the patients with acute respiratory symptoms although the findings of vital signs or physical examinations were normal. The implementation of this prediction rule can significantly decrease the costs associated with these unnecessary radiographs. In fact, its application can save time, increase clinicians' efficiency, and decrease radiation exposure to the population.

Approximately $6 \%$ of the cases would be dismissed by following this prediction rule; for instance, patients with normal findings in vital signs assessment or physical examinations who have positive results for CXR. Although this might be acceptable, it is only reasonable to implement the prediction rule for patients with reliable followups and a low likelihood of morbidity if CAP is not initially detected.

There are some limitations and shortcomings in our study. The first limitation was that performing radiographs for the study population was determined by the clinicians' judgment (selection bias); thus, our results would best reflect the current medical practices, which normally start by a visit from general practitioners. The second limitation was interpreting equivocal CXR findings as positive; however, this classification minimized the false negative results. Finally, the inevitable delay between the onset of symptoms and their appearance on CXR was not explored in this study and the insignificant findings on CXR could be partly related to this issue. The prediction rule of our study must be independently validated and until then, the results should be interpreted with caution.

Our findings suggested that it might not be necessary to perform CXR in patients with acute respiratory symptoms unless the vital signs and/or physical examination findings are abnormal. However, since the sensitivity of this rule is $94 \%$ for predicting CAP, a CXR is required for patients with unreliable follow-ups or moderate to high likelihood of morbidity if CAP is not initially detected.

\section{Authors' Contributions}

Azadeh Ebrahimzadeh: final approval of the article. Mahyar Mohammadifard: critical revision and final ap proval of the article for important intellectual content. Godratallah Naseh: drafting of the article. Alireza Mirgholami: collection and assembly of data.

\section{Funding/Support}

This study was supported by the Faculty of Medicine and the Chancellery for Research of Mashhad University of Medical Sciences.

\section{References}

1. Kumar V, Abbas AK, Fausto N, Mitchell R. Robbins Basic Pathology 8 edPhiladelphia: Elsevier Health Sciences; 2007.

2. Mardani M, Keshtkar Jahromi M, Mir Eshghi M. Evaluating Infectious Etiologies of Hospitalization in Elderly Population of Iran. Middle East J Age Aging. 2006;3(2).

3. Mamishi S, Eghbali AN, Rezaei N, Abolhassani H, Parvaneh N, Aghamohammadi A. A single center 14 years study of infectious complications leading to hospitalization of patients with primary antibody deficiencies. Braz J Infect Dis. 2010;14(4):351-5.

4. Metlay JP, Kapoor WN, Fine MJ. Does this patient have community-acquired pneumonia? Diagnosing pneumonia by history and physical examination. JAMA. 1997;278(17):1440-5.

5. Heckerling PS. The need for chest roentgenograms in adults with acute respiratory illness. Clinical predictors. Arch Intern Med. 1986;146(7):1321-4.

6. Levy ML, Le Jeune I, Woodhead MA, Macfarlaned JT, Lim WS, British Thoracic Society Community Acquired Pneumonia in Adults Guideline G. Primary care summary of the British Thoracic Society Guidelines for the management of community acquired pneumonia in adults: 2009 update. Endorsed by the Royal Col- 
lege of General Practitioners and the Primary Care Respiratory Society UK. Prim Care Respir J. 2010;19(1):21-7.

7. Ayalon I, Glatstein MM, Zaidenberg-Israeli G, Scolnik D, Ben Tov A, Ben Sira L, et al. The role of physical examination in establishing the diagnosis of pneumonia.Pediatr Emerg Care. 2013;29(8):893-6.

8. Metlay JP, Fine MJ. Testing strategies in the initial management of patients with community-acquired pneumonia. Ann Intern Med. 2003;138(2):109-18.

9. Diehr P, Wood RW, Bushyhead J, Krueger L, Wolcott B, Tompkins RK. Prediction of pneumonia in outpatients with acute cough-a statistical approach.J Chronic Dis. 1984;37(3):215-25.

10. Singal BM, Hedges JR, Radack KL. Decision rules and clinical prediction of pneumonia: evaluation of low-yield criteria. Ann Emerg Med. 1989;18(1):13-20.

11. Gennis P, Gallagher J, Falvo C, Baker S, Than W. Clinical criteria for the detection of pneumonia in adults: guidelines for ordering chest roentgenograms in the emergency department. J Emerg Med.1989;7(3):263-8.

12. O'Brien WT, Sr., Rohweder DA, Lattin GE, Jr., Thornton JA, Dutton JP, Ebert-Long DL, et al. Clinical indicators of radiographic findings in patients with suspected community-acquired pneumonia: who needs a chest x-ray? J Am Coll Radiol. 2006;3(9):703-6.

13. Graffelman AW, le Cessie S, Knuistingh Neven A, Wilemssen FE, Zonderland HM, van den Broek PJ. Can history and exam alone reliably predict pneumonia? J Fam Pract. 2007;56(6):465-70.

14. Hopstaken RM, Muris JW, Knottnerus JA, Kester AD, Rinkens PE, Dinant GJ. Contributions of symptoms, signs, erythrocyte sedimentation rate, and C-reactive protein to a diagnosis of pneumonia in acute lower respiratory tract infection. Br J Gen Pract. 2003;53(490):358-64.

15. Lieberman D, Shvartzman P, Korsonsky I, Lieberman D. Diagnosis of ambulatory community-acquired pneumonia. Comparison of clinical assessment versus chest X-ray. Scand J Prim Health Care. 2003;21(1):57-60.

16. Aagaard E, Maselli J, Gonzales R. Physician practice patterns: chest $x$-ray ordering for the evaluation of acute cough illness in adults. Med Decis Making. 2006;26(6):599-605.

17. Nolt BR, Gonzales R, Maselli J, Aagaard E, Camargo CA, Jr., Metlay JP. Vital-sign abnormalities as predictors of pneumonia in adults with acute cough illness. Am JEmerg Med. 2007;25(6):631-6.

18. Butcher BL, Nichol KL, Parenti CM. High yield of chest radiography in walk-in clinic patients with chest symptoms.J Gen Intern Med.1993;8(3):115-9.

19. Mandell LA, Wunderink RG, Anzueto A, Bartlett JG, Campbell GD, Dean NC, et al. Infectious Diseases Society of America/American Thoracic Society consensus guidelines on the management of community-acquired pneumonia in adults. Clin Infect Dis. 2007;44 Suppl 2:S27-72. 